\title{
Bioremediation: a green approach for restoration of polluted ecosystems
}

\author{
Naveen Kumar Arora ${ }^{1}$ \\ Published online: 23 November 2018 \\ (c) Society for Environmental Sustainability 2018
}

Anthropogenic activities have resulted in deterioration of ecosystems throughout the globe making them unfit for survival of indigenous biological forms. This has also put a huge pressure on the fast receding natural resources. Environmental pollution has been a major concern over the past few decades influencing the quality of life. Rampant industrialization, improper agricultural methods, unchecked discharge of pollutants into land and water bodies has severely contaminated the ecosystems on earth. This has resulted in inadequate use of natural resources, increase in barren fields, loss of biodiversity, problem of potable water and huge economic losses which are very difficult to even estimate. Manmade chemicals are increasing by the day and many of them are recalcitrant and most being xenobiotic. As per estimates every year 10 million tons of toxic chemicals are released into the environment around the globe. Due to the addition of dangerous toxic chemicals such as polycyclic aromatic hydrocarbons (PAH), polychlorinated biphenyls (PCBs), soil and water systems have become contaminated. These pollutants are carcinogenic and are persistent, causing great harm to the ecosystems harming the health of the environment and causing damage to all life forms. Heavy metal pollution is another baleful influence of human activity on environment. Industrial processes including refineries, metal processing units, waste incineration, fossil fuel burning, nuclear power plants, lead based paints, plastics, electronic wastes, agrochemicals, pharmaceutical chemicals, vehicle exhausts, leather industries are the major sources of heavy metal contamination in the environment. Heavy metals degrade the quality of soil and water, rendering them unsustainable and of little or no use, also negatively impacting the biological health of ecosystems. The green revolution in 1950s or 60s although helped in increasing food productivity but caused

Naveen Kumar Arora

nkarora.bbau@gmail.com

1 Department of Environmental Science, BBA University, Lucknow, UP, India acute toxification of the environment due to the injudicious use of chemical pesticides and fertilizers. Recalcitrant nature, particularly of the pesticides has resulted in their long term accumulation in soil, water and food chains. As per the report of Food and Agriculture Organization (FAO) production of pesticides has increased from 0.2 million tons in 1950 to 5 million tons in 2000 resulting in deterioration of fertile land, killing non-targeted microbes, birds, animals and proving fatal for human health. World Health Organization (WHO) estimates that pesticides cause quarter of a million premature deaths and over 3 million people are being hospitalized each year due to poisoning. Oil spills in oceans and on land have also played havoc with the respective ecosystems endangering the life and quality of natural systems to a great extent. In last 5 years 40,000 tonnes of oil was estimated to be spilled in the marine ecosystems through offshore mining, accidents, damaged tankers etc. The insoluble oil strata cuts off the oxygen supply and sunlight from entering the water body causing the death of flora and fauna, extinction of species and reduced microbial population. On land spilled oil forms a layer, impacting the oxygen levels and releasing toxic components, rendering the system non-fertile.

Apart from these, climate change caused by ever-increasing emission of green house gases (GHGs), has resulted in degradation of ecosystems due to rising temperature, droughts, floods etc. Incidences of heavy or very low rains have become common now throughout the globe causing flash floods or drought. Very recent floods in Kerala, India, one of the biggest in last 100 years in the region, have completely devastated the agricultural fields and yields due to accumulation of silt and washing off of the top soil. Similarly, wild fires in California have resulted in great loss to the forest, ecosystem of the region and also to human life, rendering the soil and the region greatly impacted. Incidences of heat waves in Europe and other parts of the world are now much more common. The impact of climate change and addition of chemicals has resulted in desertification and 
salinization of land resulting in lower productivity and lesser availability of biological resources. Approximately one billion hectares of land is suffering from the problem of salinity around the globe and this menace is increasing by the day. Water bodies including rivers, lakes or oceans have been impacted, causing great loss to their biodiversity or utility. With increasing human population we need more and more resources, productive fields, clean water bodies so as to get the maximum out of the natural resources. For this we have to clean-up the mess and remove toxic pollutants from the ecosystems, reclaim waste and marginal lands, saline soils, rejuvenate fresh water bodies and make oceans free of contaminants.

Over the years, various conventional methods like physical, chemical and thermal processes have been used to clean and remediate the ecosystems. But there have been several and some serious drawbacks associated with these processes, such as production of toxic intermediates, transport of contaminated soil/ water for treatment, high costs of treatment and inefficient revival of natural flora and fauna. However, use of biodegradation and bioremediation techniques involving biological systems such as microorganisms or their products and plants, are sustainable, cost effective options abating and rendering the pollutants harmless by natural biological activities. In bioremediation biological systems are applied for reclamation of the contaminated soil/ water by transformation of toxic pollutants into less hazardous or completely non-hazardous forms. The bioremediation technology includes extensive use of microorganisms or their enzymatic machineries, phytoremediation (plants) and rhizoremediation (plant and microbe interactions) techniques. Bioremediation is of two types depending on their site of application including cheaper and much effective in situ remediation where pollutants are treated on the site of contamination and the ex situ remediation where the contaminated samples are brought to laboratory and industries and are treated; used more for treatment of highly polluted but for smaller area or for systems such as ground water, where it is not advisable to add microbes or their enzymes. Technical aspects of bioremediation involve various mechanisms such as biosequestration, biodegradation, phytohydraulics, biological extraction, and volatilization by which microbes or plants immobilize or transform the complex moieties of the pollutants remediating the land and water. These biological systems have successfully been applied in cleaning up of ground water, lagoons, sludge, water streams, agricultural lands, oil spills, petroleum and hydrocarbon contaminated sites. Bioremediation through microorganisms generally involves the application of aerobic bacteria reported to degrade pesticides and hydrocarbons, both alkanes and polyaromatic compounds and several other pollutants. Anaerobic bacteria are used for bioremediation of PCBs in river sediments and dechlorination of trichloroethylene (TCE), and chloroform; lignolytic fungi for degradation of toxic and persistent pollutants; methylotrophs for remediation of chlorinated aliphatics trichloroethylene and 1,2-dichloroethane and so on. Large scale treatment of petroleum hydrocarbon contamination in oceans, waste water treatment in polar regions, removal of toxic pollutants from agro-industrial wastes, treatment of polluted shorelines have been reported to be successfully done through microbial based bioremediation. Heavy metal contamination from soil is being effectively removed by microbial or phytoremediation techniques. Rhizoremediation is a cheap and efficient technique useful for remediation of contaminated soils by the combined action of plant and their symbiotic rhizosphere microbes. Plant growth promoting microbes have been used for reclamation of saline or non-fertile marginal lands by enhancing crop productivity. After repeated use year after year, these microbes with symbiotic partners i.e. plants help to reclaim the barren and saline soils making them fertile. This results in bioremediation of such wastelands and also control of climate change due to increased carbon sequestration by the remediated ecosystems. Many success stories of highly and vastly contaminated sites are now known, including long shore lines, as in Alaska oil spill, or decontamination of polluted soils or agriculture fields. Genetic engineering has very important role to play in the area of bioremediation. Capable microorganisms are engineered to improve their cell membrane transportation or their enzymatic attributes supporting the enhanced and wide spectral degradation of pollutants. "Superbugs" are the most popular examples of genetically engineered biological tools, significant in remediating oil spills and other toxic pollutants. Microbes are being engineered to have genes for degradation of multiple components of the complex crude oil and there are success stories in this area. Pyrosequencing, a next-generation molecular approach, is contributing to study pollutant-microbe interactions. With the help of pyrosequencing, environmental responses of microbes to contaminants, microbiome resistance against pollutants, diversity of fungal degrading genes in soil, can be known and used much more efficiently in bioremediation of contaminated sites. Quorum sensing properties of microbes have also been implemented for bioremediation as stimulation of signaling molecules like acylated homoserine lactone (AHL) also regulate gene expression for exopolysaccharides (EPS) and biofilm formation helping in degradation. Microbial fuel cells, microbial electrolysis cells and microbial desalination cells, bioelectrical wells and biofiltration are other advanced biotechnological approaches used for removal of various toxicants from the environment. Exploitation of effective genes from non-culturable microbes in a contaminated ecosystem can be used for degradation and remediation of complex or multiple polluted sites. Metagenomics, transcriptomics, metabolomics and fluxomics can play very important roles in future to identify the important 
genes involved in biodegradation, their expression, release of important enzymes and rates of metabolic reaction even in highly contaminated ecosystems. This can go a long way in improving the quality of stressed ecosystems, because most of them have huge populations of non-culturable microbes with great genetic pool and abilities to survive and flourish in such habitats. Need is to exploit them by the process of bioaugmentation of nutrients or through co-metabolism. Hence, although we have already achieved a bit in the area of bioremediation but a lot is yet to be explored and future of this green technology in cleaning up the environment is very bright.

Bioremediation has a great potential with notable achievements already reported from around the globe. But still this excellent and eco-friendly low input biotechnology has been underutilized. The global market scenario of bioremediation technology and services is showing an elevation with compound annual growth rate of $8.3 \%$ from 2017 to 2025 . This can be much higher if exploited and developed properly. Bioremediation and biodegradation are the key focus areas of the journal "Environmental Sustainability". We need to clean the mess created by anthropogenic activities through green technologies so as to provide a healthy and sustainable planet to the future generations. 